\title{
GALQR Optimal Control Method and Applying in the Active Suspension System
}

\author{
Guosheng Zhang Zongde Fang Lei Shu \\ School of Mechatronic Engineering, Northwestern Polytechnical University, Xi'an 710072, P. R. China
}

\begin{abstract}
This paper adds genetic algorithm (GA) to Linear Quadratic Regulator (LQR) and presents a new optimal control method-GALQR. The GALQR control method uses GA to optimize the weight matrices of LQR and adopts the divisional removing searching mechanism to insure getting the global optimal results. The method was applied to design the control schedules of the Active Suspension System. Comparing with the experiential LQR, the results show the GALQR can increase the possibility of finding the optimal weight matrices and make the system gain better design performances.
\end{abstract}

Keywords: Genetic algorithm, LQR, Optimization, Weight, Active suspension system

\section{Introduction}

Linear Quadratic Regulator (LQR) is an optimal control method with the quadratic performance indexes and these indexes have specific physical concepts generally [1]-[2]. At the same time, LQR has simple math disposal process and can achieve closed loop optimal control with the linear state feedback or output feedback [3]-[5]. LQR has been applied in engineering widely [6]-[10].

The selection of weight matrices in LQR is very important and it straight affects the control effect. In general, the weight matrices are set by engineers, who need be familiar with the controlled system [11]-[13]. Reference [14] applied LQR to design the control law of the Active Suspension System (ASS). The weight matrices were set by experience first and then were adjusted by simulation till obtaining the satisfying output responses. To this process, if the designer known poor about the system, the optimal weight matrices could not be obtained and so the control performances also could not be optimal.

The genetic algorithm (GA) is a highly efficient and robust search algorithm based on evolution in nature [15]-[17]. GA has been widely used to evolve good solutions to hundreds of different problems [18][20].
The purpose of this paper is to propose a new optimal control method, which is called GALQR (Genetic Algorithm Linear Quadratic Regulator), through adding the GA to the LQR for optimizing the weight matrices of LQR. GALQR is expected to overcome the shortcoming of general LQR. The paper is organized as follows. In section 2 , the concept of LQR optimal control method is expounded. In section 3 , the idea of GALQR design method is illuminated and the steps for realizing GALQR are introduced. In section 4, the GALQR is applying to the design of control laws for the ASS. In section 5, the contrasting results of the optimization in two different methods (the GALQR and the experiential LQR) are presented. In section 6 , some concludes are given.

\section{Lqr optimal control}

The LQR is an optimal control method for the linear system. In this section, the basic design process for LQR will be illuminated.

Suppose the state equation of the controlled system is as follow:

$$
\left\{\begin{array}{l}
\dot{\boldsymbol{x}}(t)=\boldsymbol{A} \boldsymbol{x}(t)+\boldsymbol{B u}(t) \\
\boldsymbol{y}=\boldsymbol{C x}(t)+\boldsymbol{D u}(t) \\
\boldsymbol{x}\left(t_{0}\right)=\boldsymbol{x}_{0}
\end{array}\right.
$$

and the performance index is given by:

$$
J=\frac{1}{2} \int_{0}^{\infty}\left[\begin{array}{l}
\boldsymbol{x} \\
\boldsymbol{u}
\end{array}\right]^{\mathrm{T}}\left[\begin{array}{cc}
\boldsymbol{Q} & \boldsymbol{N} \\
\boldsymbol{N}^{\mathrm{T}} & \boldsymbol{R}
\end{array}\right]\left[\begin{array}{l}
\boldsymbol{x} \\
\boldsymbol{u}
\end{array}\right] \mathrm{d} t
$$

where $\boldsymbol{Q}, \boldsymbol{R}$ and $N$ are the weigh matrices, $\boldsymbol{Q}$ is required to be positive definite or positive semi-definite symmetry matrix, $N$ is required to be positive definite symmetry matrix.

If the system described by (1) is controllable completely, the control method which makes (2) achieving minimum is called Linear Quadratic Regulator.

Using (2), the correctional Riccati matrix equation can be obtained:

$$
\boldsymbol{P A}+\boldsymbol{Q A} A^{\mathrm{T}} \boldsymbol{P}-(\boldsymbol{P B}+N) N R^{-1}(P B+N)^{\mathrm{T}}+Q=0
$$


By solving (3), matrix $\boldsymbol{P}$ can be obtained and if it is positive definite, the system will be steady and the optimal feedback vector $\boldsymbol{K}$ and the optimal control variable $\boldsymbol{u}(\boldsymbol{t})$ are gained:

$$
\begin{aligned}
& \boldsymbol{K}=\boldsymbol{R}^{-1}\left(\boldsymbol{B}^{\mathrm{T}} \boldsymbol{P}+\boldsymbol{N}^{\mathrm{T}}\right) \\
& \boldsymbol{u}(\boldsymbol{t})=-\boldsymbol{K} \boldsymbol{x}(\boldsymbol{t})
\end{aligned}
$$

For LQR control problem, the weigh matrices in the performance index have a great influence on the control effects. In general designs, the weigh matrices are set based on the physical process by experience. In order to achieve the better control performances, the engineer need be very familiar with the controlled system.

\section{Galqr optimal control method}

In this section, GA will be used to optimize the weight matrices of LQR and the optimal control method is called GALQR. The main idea of the GALQR is to utilize the random searching ability of the GA to find the optimal weight matrices and so the improvements of the design efficiency and the design performances are expected.

The GALQR retains the basic structure of the GA: coding, selection, crossover, mutation. For the problem in this paper, whereas the value ranges for weight matrices $\boldsymbol{Q}$ and $\boldsymbol{R}$ are quite wide, the divisional removing searching mechanism is adopted in the general GA to enhance the searching efficiency and the searching precision.

The basic ideas for the divisional removing searching mechanism: the searching space is divided into some small searching spaces (the amount of the small searching spaces is set based on the searching efficiency and the searching precision), and then the GA operation with constraints is applied in every small searching space. The space in which there is no chromosome satisfying constraints to be found within appointed searching times will be removed.

The basic design process for the GALQR optimal control method is as follows:

Step 1. Coding: The binary code is adopted and the main aim of the coding is to obtain the binary code digit correspond to the decimal parameter. For the parameters of weigh matrices $\boldsymbol{Q}$ and $\boldsymbol{R}$, the corresponding binary code digits are gained according to the ranges and the precision demands of the parameters. Suppose the range of parameter $q_{i}$ is $\left[L_{q_{i}}, U_{q_{i}}\right]$ and the precision demand is $\varepsilon$, then the binary code digit $B_{q_{i}}$ for $q_{i}$ is obtained as follow:

$$
B_{q_{i}}=\left[\log _{2}\left(\left(U_{q_{i}}-L_{q_{i}}\right) / \varepsilon\right)\right]_{+\infty}
$$

When the binary code digits for all parameters have been obtained using (6), the whole chromosome digit $B$ is obtained:

$$
B=\sum_{i=1}^{n_{1}} B_{q_{i}}+\sum_{j=1}^{n_{2}} B_{r_{j}}
$$

where $[\cdot]^{+\infty}$ denotes rounding to positive infinitude, $B_{q_{i}}$ $\left(i=1 \sim n_{1}\right)$ and $B_{r_{j}}\left(j=1 \sim n_{2}\right)$ are the binary codes for the weigh matrix $\boldsymbol{Q}$ and $\boldsymbol{R}$ respectively, $n_{1}$ and $n_{2}$ are the amount of parameters included in $\boldsymbol{Q}$ and $\boldsymbol{R}$ respectively.

Step 2. Dividing Searching Space: Here will bring the GA with two parameters as an example to illuminate the process for dividing. Suppose the range of parameter $q_{i}$ is $\left[L_{q_{i}}, U_{q_{i}}\right]$ and the divided space number is $m_{q_{i}}$; the range of parameter $q_{j}$ is $\left[L_{q_{j}}, U_{q_{j}}\right]$ and the divided space number is $m_{q_{j}}$. Then the whole searching space is divided into $m_{q_{i}} \cdot m_{q_{j}}$ small searching spaces. For the amount of small searching spaces is increasing by times with the number of parameters, the searching time also will be increasing by times. So the GALQR will be applied in the design problem with the number of the design parameter under four.

The small spaces are numbered and the searching domain numbers for all parameters in every small searching space are registered in a matrix, e.g. the matrix $\boldsymbol{M}$ below is for the problem with two parameters above.

$$
\boldsymbol{M}=\left[\begin{array}{ccc}
1 & 1 & 1 \\
2 & 1 & 2 \\
\vdots & \vdots & \vdots \\
k & k_{q_{i}} & k_{q_{j}} \\
\vdots & \vdots & \vdots \\
m_{q_{i}} \cdot m_{q_{j}} & m_{q_{i}} & m_{q_{j}}
\end{array}\right]
$$

Thereinto, the first column is the numbers of the searching spaces and the second and the third column are the searching domain numbers of the parameter $q_{i}$ and $q_{j}$ respectively.

According to the matrix $\boldsymbol{M}$, in the $k^{\text {th }}$ searching space, the searching domain numbers of the parameter $q_{i}$ and $q_{j}$ are $k_{q_{i}}$ and $k_{q_{j}}$ respectively. Thereby, the value domain in the $k^{\text {th }}$ searching space for the parameter $q_{i}$ is counted as following equations:

$$
\begin{aligned}
& L_{q_{i}}^{k_{q_{i}}}=L_{q_{i}}+\frac{U_{q_{i}}-L_{q_{i}}}{m_{q_{i}}} \cdot\left(k_{q_{i}}-1\right) \\
& U_{q_{i}}^{k_{q_{i}}}=L_{q_{i}}+\frac{U_{q_{i}}-L_{q_{i}}}{m_{q_{i}}} \cdot k_{q_{i}}
\end{aligned}
$$

where $L_{q_{i}}^{k_{q_{i}}}$ and $U_{q_{i}}^{k_{q_{i}}}$ are the lower limit and the upper limit of value domain of the parameter $q_{i}$ in the $k^{\text {th }}$ 
searching space. The count for the value domain of the parameter $q_{j}$ is just the same.

Step 3.Creating initial Group: In the $i^{\text {th }}$ searching space, suppose the scale of the group is $P_{\mathrm{s}}$ and the whole chromosome digit is $B$, then the $P_{\mathrm{s}}$ individual chromosomes in the form of the binary code will be produced as an initial group using the random method.

Step 4.Checking Design Constraints: Al chromosomes in the $i^{\text {th }}$ searching space will be checked to ensure if they satisfy the design constraints. Here carries out the Step3 and step4 three times for the exact judgment. Through the constraint checking, if there is no chromosome satisfying constraints to be found in the searching space, the removing sign will be set and the operation will transfer to Step8, or transfer to Step5.

Step 5.Selection: For chromosomes satisfying the constraints in the $i^{\text {th }}$ searching space, the fitness selection principle is adopted to obtain next generation chromosomes. To the LQR problem, the fitness selection principle is as follow:

First, the decode operation which transforms the binary code to the decimal code will be applied to chromosome $b_{i}$. Suppose $b_{i}$ is composed of $N$ design variables and the $k^{\text {th }}$ variable contains $n_{k}$ binary bits, and its decimal value range is $\left[L_{b_{i}}^{k}, U_{b_{i}}^{k}\right]$, then the decode operation for the $k^{\text {th }}$ variable is as follow:

$$
q_{b_{i}}^{k}=L_{b_{i}}^{k}+\left(\sum_{i=1}^{n_{k}} 2^{i-1} K_{i-1}\right) \cdot \frac{U_{b_{i}}^{k}-L_{b_{i}}^{k}}{2^{n_{k}}-1}
$$

where $K_{i}$ is the $i^{\text {th }}$ code digit of the $k^{\text {th }}$ variable. Using (9), all parameters of weigh matrices $\boldsymbol{Q}$ and $\boldsymbol{R}$ can be decoded.

Next, through solving (3), the optimal feedback vector $\boldsymbol{K}$ and the optimal control variable $\boldsymbol{u}(\mathrm{t})$ can be obtained, and then the closed loop optimal control system can be formed by the state linear feedback and the output $x(t)$ is also obtained.

Suppose the fitness function of GA is denoted by $f_{g a}=f(b)$ and the fitness for the chromosome $b_{i}$ is $f\left(b_{i}\right)$, when the number of chromosomes satisfying the constraints is $N_{\mathrm{c}}$, then the reproduced number for the chromosome $b_{i}$ is counted as follow:

$$
P\left\{b_{i}\right\}=\frac{f\left(b_{i}\right)}{\sum_{j=1}^{N_{c}} f\left(b_{j}\right)} \cdot N_{\mathrm{c}}
$$

Equation (10) shows that the reproduced number of the chromosome is in direct proportion to its fitness.

Step 6.Crossover: Crossover operation will face to the all chromosomes in the $i^{\text {th }}$ searching space. During the crossover operation, the position for crossover is random selected and the operation is carried out between two selected chromosomes by crossover probability $P_{c}$.
Step 7.Mutation: To the group produced by the crossover operation, according to the gene mutation principle in the biology, the mutation operation is carried out at some bits in the selected chromosomes by mutation probability $P_{\mathrm{m}}$. During the operation, the bit required to mutate is reversed: 1 to 0 and 0 tol. In order to increase the global search ability of GA, here the mutation probability $P_{\mathrm{m}}$ will be increasing along with the evolution process:

$$
P_{\mathrm{m}}=P_{\mathrm{m}_{0}}+\frac{E_{\mathrm{n}}^{4}\left(0.6-P_{\mathrm{m}_{0}}\right)}{E_{\mathrm{m}}^{4}}
$$

where $P_{\mathrm{m}_{0}}$ is the initial mutation probability, $E_{\mathrm{m}}$ is the total epoch, $E_{\mathrm{n}}$ is the current evolution epoch.

Step 8. If the searching space number $i$ is less than the current total space number, $i=i+1$ and the operation will transfer to Step3, or transfer to Step9.

Step 9.Judging to Stop: If the current evolution epoch is less than the total epoch, the space with the removing sign will be eliminated from the searching domain and the total space number is updated. At the same time, the number $i$ is set to be 1 and the operation will transfer to step3, or stop the GALQR operation and output the results.

Fig. 1 is corresponding to the theory of GALQR above. From the process of realizing GALQR, it is shown that the space dissatisfying the constraints will not be contained in the next circle recurring to the divisional removing searching mechanism and so GALQR has the ability of globally wide search and locally careful search. Thereby, the GALQR can ensure to obtain the optimal weight matrices and make the controlled system get the better performances. In the next section, the GALQR control method will be applying to the control law design for the ASS and the superiorities will be shown.

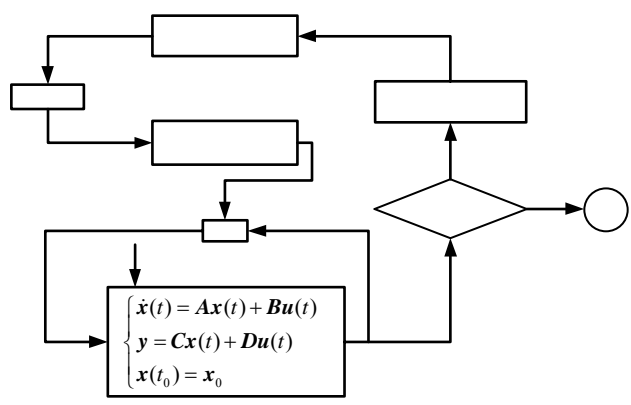

Fig. 1: Process of GALQR optimal control.

\section{Applying of galqr in the active suspension system}




\subsection{Dynamic model for the active suspension system}

In this paper, the quarter vehicle model is adopted (see Fig. 2) and the dynamical model is as follow [21]:

$$
\begin{aligned}
& m_{\mathrm{b}} \ddot{x}_{\mathrm{b}}=k_{\mathrm{s}}\left(x_{\mathrm{w}}-x_{\mathrm{b}}\right)+c_{\mathrm{s}}\left(\dot{x}_{\mathrm{w}}-\dot{x}_{\mathrm{b}}\right)+U \\
& m_{\mathrm{w}} \ddot{x}_{\mathrm{w}}=k_{\mathrm{t}}\left(x_{\mathrm{r}}-x_{\mathrm{w}}\right)-k_{\mathrm{s}}\left(x_{\mathrm{w}}-x_{\mathrm{b}}\right)-c_{\mathrm{s}}\left(\dot{x}_{\mathrm{w}}-\dot{x}_{\mathrm{b}}\right)-U
\end{aligned}
$$

where $m_{\mathrm{b}}$ is the mass of the quarter vehicle, $m_{\mathrm{w}}$ is the unsprung mass, $x_{\mathrm{b}}$ is the displacement of the vehicle body, $x_{\mathrm{w}}$ is the displacement of the wheel, $x_{\mathrm{r}}$ is the disturbance of the road; $k_{\mathrm{s}}$ and $c_{\mathrm{s}}$ are the stiffness and damp of the suspension, $k_{\mathrm{t}}$ is the stiffness of the wheel, $\mathrm{U}$ is the control force.

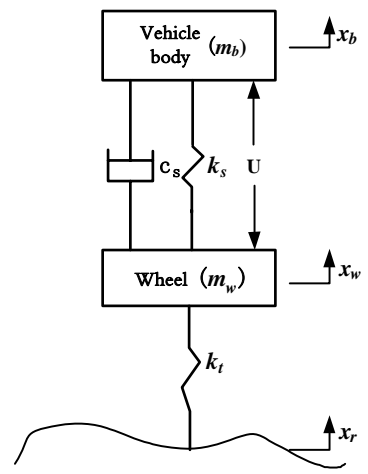

Fig. 2: Quarter vehicle model.

Select the state variable $\boldsymbol{x}$ as $\left[\dot{x}_{\mathrm{b}}, \dot{x}_{\mathrm{w}}, X_{\mathrm{b}}-X_{\mathrm{w}}, X_{\mathrm{w}}-X_{\mathrm{r}}\right]^{\mathrm{T}}$, the control variable $\boldsymbol{u}$ as control force $U$ and the disturbance $\boldsymbol{w}$ as velocity disturbance $\dot{x}_{\mathrm{r}}$ of the road, then the state equation of the system can be obtained:

$$
\begin{aligned}
& \dot{x}=A x+B u+G w \\
& y=C x+D u
\end{aligned}
$$

where, $\boldsymbol{A}=\left[\begin{array}{cccc}-\frac{c_{\mathrm{s}}}{m_{\mathrm{b}}} & \frac{c_{\mathrm{s}}}{m_{\mathrm{b}}} & -\frac{k_{\mathrm{s}}}{m_{\mathrm{b}}} & 0 \\ \frac{c_{\mathrm{s}}}{m_{\mathrm{w}}} & -\frac{c_{\mathrm{s}}}{m_{\mathrm{w}}} & \frac{k_{\mathrm{s}}}{m_{\mathrm{w}}} & -\frac{k_{\mathrm{t}}}{m_{\mathrm{w}}} \\ 1 & -1 & 0 & 0 \\ 0 & 1 & 0 & 0\end{array}\right] \quad \boldsymbol{B}=\left[\begin{array}{c}\frac{1}{m_{\mathrm{b}}} \\ -\frac{1}{m_{\mathrm{w}}} \\ 0 \\ 0\end{array}\right]$

$$
\boldsymbol{G}=\left[\begin{array}{c}
0 \\
0 \\
0 \\
-1
\end{array}\right] \quad \boldsymbol{C}=\left[\begin{array}{cccc}
-\frac{c_{\mathrm{s}}}{m_{\mathrm{b}}} & \frac{c_{\mathrm{s}}}{m_{\mathrm{b}}} & -\frac{k_{\mathrm{s}}}{m_{\mathrm{b}}} & 0 \\
0 & 0 & 1 & 0 \\
0 & 0 & 0 & 1
\end{array}\right] \quad \boldsymbol{D}=\left[\begin{array}{c}
1 \\
m_{\mathrm{b}} \\
0 \\
0
\end{array}\right]
$$

It can be proved that the system described by (13) is controllable [22] and so GALQR can be used in this system. In the next step, we will design the control law for the Active Suspension System using GALQR.

\subsection{Optimal Control Design for the ASS Using GALQR}

Main performances for evaluating the comfort and the security of a vehicle are the vertical acceleration of the vehicle body, the dynamic deflection of the suspension and the dynamic deflection of the tire. At the same time, in order to decrease the energy depletion of the ASS, the control force input also is taken into account. Thereby, the quadratic performance index is selected as:

$$
J=\frac{1}{2} \int_{0}^{\infty} q_{1} \ddot{x}_{\mathrm{b}}^{2}+q_{2}\left(x_{\mathrm{b}}-x_{\mathrm{w}}\right)^{2}+q_{\mathrm{3}}\left(x_{\mathrm{w}}-x_{\mathrm{r}}\right)^{2}+R_{\mathrm{l}} u^{2} \mathrm{~d} t
$$

Using (13), equation (14) can be transformed into following form:

$$
J=\frac{1}{2} \int_{0}^{\infty}\left[\boldsymbol{x}^{\mathrm{T}} \boldsymbol{Q} \boldsymbol{x}+u^{\mathrm{T}}\left(\boldsymbol{R}_{1}+\boldsymbol{R}_{2}\right) u+\boldsymbol{x}^{\mathrm{T}} \boldsymbol{N} u+u^{\mathrm{T}} \boldsymbol{N}^{\mathrm{T}} \boldsymbol{x}\right] \mathrm{d} t
$$

where $\boldsymbol{Q}=\boldsymbol{C}^{\mathrm{T}} \boldsymbol{Q}_{\mathrm{p}} \boldsymbol{C}, \boldsymbol{R}_{2}=\boldsymbol{D}^{\mathrm{T}} \boldsymbol{Q}_{\mathrm{p}} \boldsymbol{D}, \boldsymbol{N}=\boldsymbol{C}^{\mathrm{T}} \boldsymbol{Q}_{\mathrm{p}} \boldsymbol{D}, \boldsymbol{Q}_{\mathrm{p}}=\operatorname{diag}\left(q_{1}, q_{2}\right.$, $\left.q_{3}\right), \boldsymbol{R}_{1}=1, \boldsymbol{R}_{=} \boldsymbol{R}_{1}+\boldsymbol{R}_{2}$

It is shown that if matrix $\boldsymbol{Q}_{\boldsymbol{p}}$ has been obtained, the weigh matrix $\boldsymbol{Q}, \boldsymbol{R}, \boldsymbol{N}$ and the matrix $\boldsymbol{P}$ can be calculated using (15) and (3) separately. Thereby, the optimal feedback vector $\boldsymbol{K}$ and the optimal control force $U$ are gained using (4) and (5).

Here, the optimized parameters are selected as $q_{1}, q_{2}$ and $q_{3}$ and the performance evaluation function is as follow:

$$
f_{\mathrm{ev}}=\frac{\psi_{1}}{\psi_{1}^{\mathrm{b}}}+\frac{\psi_{2}}{\psi_{2}^{\mathrm{b}}}+\frac{\psi_{3}}{\psi_{3}^{\mathrm{b}}}
$$

where $\psi_{1}, \psi_{2}$ and $\psi_{3}$ are the Root Mean Square (RMS) of the vertical acceleration of the vehicle body, the dynamic deflection of the suspension and the dynamic deflection of the tire for the ASS separately. $\psi_{1}^{\mathrm{b}}, \psi_{2}^{\mathrm{b}}$ and $\psi_{3}^{\mathrm{b}}$ are the RMS of the Passive Suspension System (PSS) relative to $\psi_{1}, \psi_{2}$ and $\psi_{3}$ separately.

From (16), it is shown that the evaluation function is standardized by the RMS of PSS, which makes the amount level of every index accordant and achieves the uniform optimization for indexes. Thereby, the fitness function for GA operation can be set as $f_{\mathrm{ga}}=1 / f_{\mathrm{ev}}$.

Considering the performances of ASS must be better than PSS firstly and the dynamic deflection of the suspension is restricted by the structure of the vehicle, constraints for the divisional removing searching mechanism are selected as:

$$
\left\{\begin{array}{l}
g(1)=\psi_{1}<\psi_{1}^{\mathrm{b}} \\
g(2)=\psi_{2}<\psi_{2}^{\mathrm{b}} \\
g(3)=\psi_{3}<\psi_{3}^{\mathrm{b}} \\
g(4)=\max \left(\left|f_{\mathrm{d}}\right|\right)<f_{\text {dlim }}
\end{array}\right.
$$


where $f_{\mathrm{d}}$ is the dynamic deflection of the suspension, $f_{\text {dlim }}$ is the allowable limit of $f_{\mathrm{d}}$.

\section{Results}

Through the analysis in section 4, the model for designing the optimal control law for the ASS using the GALQR has been set up. Next, the design operation will be done and the contrasts between the GALQR and the experiential LQR will be presented.

The design parameters for the GALQR are selected as: search domains for $q_{1}, q_{2}$ and $q_{3}$ are $\left[1,10^{8}\right]$ likewise; $\varepsilon=1 \times 10^{4} ; \quad P_{\mathrm{s}}=30 ; \quad P_{\mathrm{c}}=0.7 ; \quad P_{\mathrm{m}_{0}}=0.1$. The dynamical parameters [2] of the vehicle are selected as: $m_{\mathrm{b}}=300 \mathrm{~kg} ; m_{\mathrm{w}}=49 \mathrm{~kg} ; k_{\mathrm{s}}=1.7 \times 10^{4} \mathrm{~N} / \mathrm{m} ; c_{\mathrm{s}}=1317 \mathrm{~N} \cdot \mathrm{s} / \mathrm{m} ; k_{\mathrm{t}}$ $=2 \times 10^{5} \mathrm{~N} / \mathrm{m} ; f_{\text {dlim }}=0.06 \mathrm{~m}$. The road conditions are: driving velocity is $20 \mathrm{~m} / \mathrm{s}$, the road is made up of rank $\mathrm{B}$ and $\mathrm{C}$ with the road undulation coefficient $64 \times 10^{-}$ ${ }^{6} \mathrm{~m}^{2} / \mathrm{m}^{-1}$ and $256 \times 10^{-6} \mathrm{~m}^{2} / \mathrm{m}^{-1}$ separately.

Using GALQR, the optimal weight vector is obtain ed: $q=\left[3823.3,9.9563 \times 10^{7}, 9.3502 \times 10^{7}\right]$. Comparing b etween the results by the GALQR and those by the ex periential LQR [2] is done (see Table 1).

\begin{tabular}{ccc}
\hline Object & GALQR & LQR $^{[2]}$ \\
\hline$q$ & {$\left[3823.3,9.9563 \times 10^{7}, 9.3502 \times 10^{7}\right]$} & {$\left[600,1 \times 10^{5}, 2 \times 10^{6}\right]$} \\
RMS_A $\left(\mathrm{m} / \mathrm{s}^{2}\right)$ & 0.98145 & 1.0088 \\
RMS_SD $\left(\times 10^{-2} \mathrm{~m}\right)$ & 5.6933 & 6.6953 \\
RMS_TD $\left(\times 10^{-3} \mathrm{~m}\right)$ & 7.7304 & 9.114 \\
\hline
\end{tabular}

Table 1: RMS Results by GALQR and LQR.

From Table 1, it is shown that all performance indexes have been improved using the GALQR method in contrast to the experiential LQR method. The Root Mean Square (RMS) of the vertical acceleration of the vehicle body is decreased $2.71 \%$, and the RMS of the dynamic deflection of the suspension and the RMS of the dynamic deflection of the tire are decreased $14.97 \%$ and $15.18 \%$ respectively.

The contrasts between two design methods about the Power Spectrum Density (PSD) of the vertical acceleration of vehicle body, the dynamic deflection of the suspension and the dynamic deflection of the tire are also shown in Fig. 3 to Fig. 5 separately.

From Fig. 3 to Fig. 5, it is shown that comparing with LQR, PSD values of the vertical acceleration of the vehicle body and the dynamic deflection of the suspension at the inherent frequency of the vehicle body $\left(f_{0}=1.2 \mathrm{~Hz}\right)$ have decreased distinctly, which will improve the comfort of the vehicle. The PSD value of the dynamic deflection of the tire only has a slight decrease at $f_{0}$ and it still makes an improvement on the handling and stability of the vehicle. Within the range of intermediate frequency and high frequency, PSD results between two methods almost are coincident, which shows that control performances of two methods approximately are same in this frequency range.

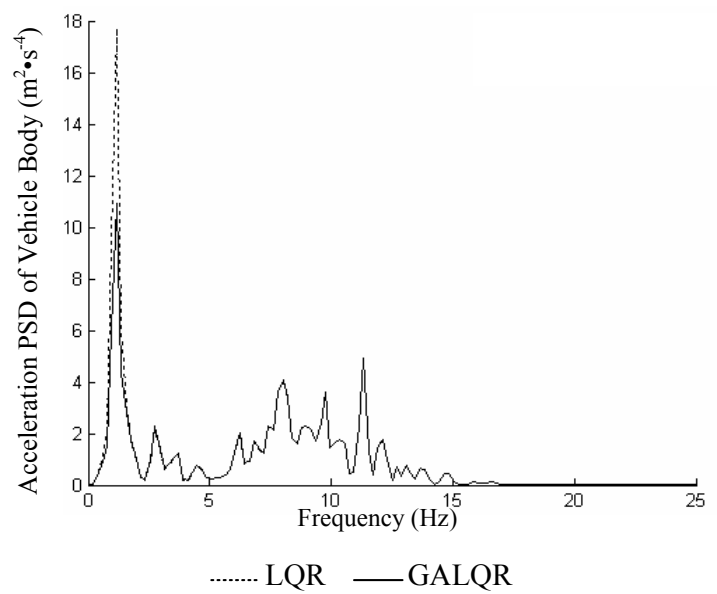

Fig. 3: Acceleration PSD of vehicle body.

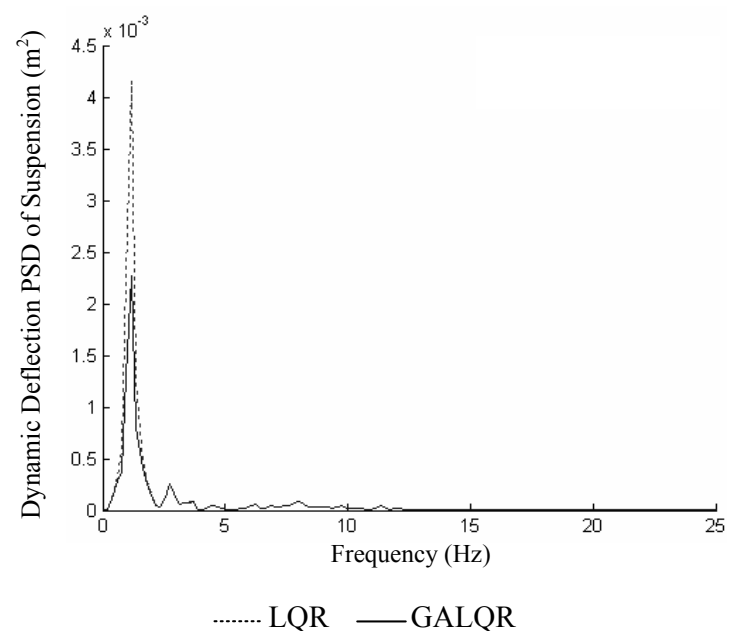

Fig. 4: Dynamic deflection PSD of suspension.

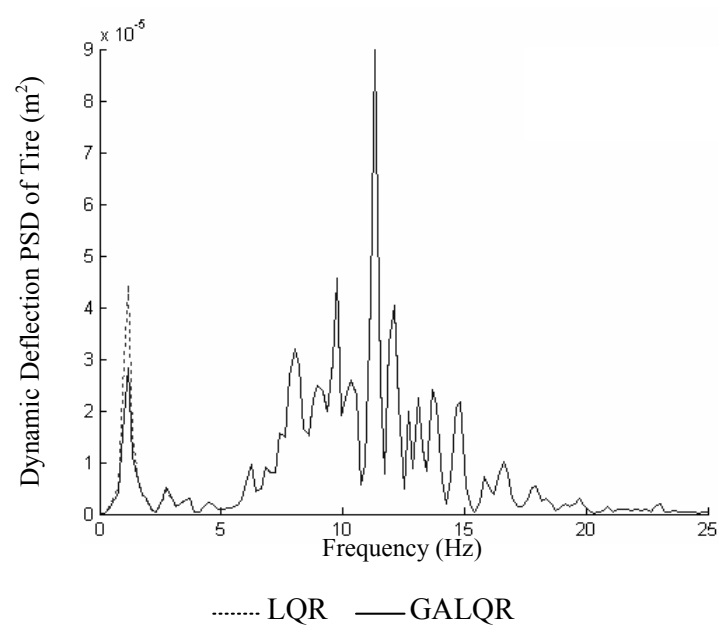

Fig. 5: Dynamic deflection PSD of tyre. 


\section{Conclusion}

In this paper, GA was added to $L Q R$ and a new optimal control method-GALQR was formed. Comparing with the experiential LQR method, GALQR method has several advantages as follows:

1) GALQR decreases the requests to the designer and the designing process is done automatically. The design efficiency is improved obviously.

2) GALQR method uses GA to optimize the weight matrices of LQR, which will increase the possibility of finding the optimal weight matrices and make the system gain better design performance.

Though only the improvement on LQR is illustrated in this paper, the design idea can be extended to other optimal control method, such as Linear Quadratic Gauss (LQG) method, which needs to be studied ulteriorly.

\section{References}

[1] H.R. H. S. Ko, K.Y. Lee and H. C. Kim, An intelligent based LQR controller design to power system stabilization, Electric Power Systems Research, 71(1):1-9, 2004.

[2] P. Zhou, F. Wang and W. Chen, PI/PID controller tuning via LQR approach, Chemical Engineering Science, 55(13): 2429-2439, 2000.

[3] P.O. M. Scokaert and J. B. Rawlings, Constrained linear quadratic regulation, IEEE Trans. Automatic Control, 43(8):1163-1169, 1998.

[4] Y. B. Shtessel, Principle of proportional damages in a multiple criteria LQR problem, IEEE Trans. Automatic Control, 41(3):461-464, 1996.

[5] P. Zhou, F. Wang and W. Chen, Optimal construction and control of flexible manipulators: a case study based on LQR output feedback, Mechatronics, 11(1):59-77, 2001.

[6] A. Meng, L. Wang and H. Cong, A study on the modeling and control strategy of automotive active suspension system, Automotive Engineering, 25(5): 474-476,513, 2003.

[7] C.P. Bottura and J. V. Da Fonseca Neto, Parallel eigenstructure assignment via LQR design and genetic algorithms, Proc. IEEE Conf. American control, pp. 2295-2299, 1999.

[8] D. Edouard, P. Dufour and H. Hammouri, Observer based multivariable control of a catalytic reverse flow reactor: comparison between LQR and MPC approaches, Computers \& Chemical Engineering, 29(4): 851-865, 2005.

[9] L. Chen, J. Zhang and G. Xi, Adaptive optimal control for automatic clutch, Journal of Shanghai Jiaotong University, 34(10):1312-1316, 2000.

[10]O. Marjanovic, B. Lennox and P. Goulding,
Minimising conservatism in infinite-horizon LQR control, Systems \& Control Letters, 46(4):271-279, 2002.

[11]B. Lincoln and B. Bernhardsson, LQR optimization of linear system switching, IEEE Trans. Automatic Control, 47(10):1701-1705, 2002.

[12]M. Aldeen and F. Crusca, Multimachine power system stabiliser design based on new LQR approach, IEE E Trans. Generation, Transmission and Distribution, 142(5): 494-502, 1995.

[13] Y. T. Choon and E. A. Misawa, Sliding surface design for discrete VSS using $\mathrm{LQR}$ technique with a preset real eigenvalue, Proc. IEEE Conf. American control, pp. 520-524, 1999.

[14]B. Dong, A study on optimal control for vehicle active suspension system using a whole vehicle model, Automotive Engineering, 24(5):422-425, 2002.

[15] M. Zhou and S. Shun, Theory and Applications of GA, National Defence Industry Press, 1999.

[16]G. Gal, S. Ronan and L. C. Laurent, Ensemble based on GA wrapper feature selection, Computers \& Industrial Engineering, 51(1):111116, 2006.

[17]Raid Al-Aomar and Ala'a Al-Okaily, A GA-based parameter design for single machine turning process with high-volume production, Computers \& Industrial Engineering, 50(3):317-337, 2006.

[18] G. Gal, S. Ronan and L. C. Laurent, Design and optimization of three-dimensional integrated lens antennas with genetic algorithm, IEEE Trans. Antennas and Propagation., 55(3):770-775, 2007.

[19]E. Dilettoso and N. Salerno, A self-adaptive niching genetic algorithm for multimodal optimization of electromagnetic devices, IEEE Trans. Magnetics., 42(4):1203-1206, 2006.

[20]E. Dilettoso and N. Salerno, A GA mechanism for optimizing the design of attribute double sampling plan, Automation in Construction, 16(3):345-353, 2007.

[21]Z. Yu, Theory of Automotive, $2^{\text {nd }} e d$, China Machine Press, 1999.

[22]D. Zheng, Linear System Theory, $2^{\text {nd }}$ ed, Tsinghua University Press, 2002. 